\title{
Treatment of hypertriglyceridemia-induced acute pancreatitis with insulin
}

\author{
Ali Coskun, Nazif Erkan, Savas Yakan, Mehmet Yildirim, Erdem Carti, Deniz Ucar, Erkan Oymaci \\ Department of General Surgery, Izmir Training and Research Hospital, Izmir, Turkey
}

Prz Gastroenterol 2015; 10 (1): 18-22 DOI: $10.5114 / p g .2014 .45412$

Key words: acute pancreatitis, hypertriglyceridemia, hyperlipidaemia, insulin.

Address for correspondence: Ali Coskun MD, Mithatpasa cad no: 964, kat 4, daire 9, Goztepe/lzmır, Turkey, phone: +90 2322850707 fax: +90 2322614444, e-mail: dralicoskun@hotmail.com

\begin{abstract}
Introduction: Hypertriglyceridaemia (HT)-induced pancreatitis rarely occurs unless triglyceride levels exceed $1000 \mathrm{mg} / \mathrm{dl}$. Hypertriglyceridaemia over $1,000 \mathrm{mg} / \mathrm{dl}$ can provoke acute pancreatitis (AP) and its persistence can worsen the clinical outcome. In contrast, a rapid decrease in triglyceride level is beneficial. Insulin-stimulated lipoprotein lipase is known to decrease serum triglyceride levels. However, their efficacy in HT-induced AP is not well documented.

Aim: To present 12 cases of AP successfully treated by insulin administration.

Material and methods: Three hundred and forty-three cases of AP were diagnosed at our clinic between 2005 and 2012. Twelve (3.5\%) of these cases were HT-induced AP. Twelve patients who suffered HT-induced AP are reported. Initial blood triglyceride levels were above $1000 \mathrm{mg} / \mathrm{dl}$. Besides the usual treatment of AP, insulin was administered intravenously in continuous infusion. The patients' medical records were retrospectively evaluated in this study.

Results: Serum triglyceride levels decreased to $<500 \mathrm{mg} / \mathrm{dl}$ within 2-3 days. No complications of treatment were seen and good clinical outcome was observed.

Conclusions: Our results are compatible with the literature. Insulin may be used safely and effectively in HT-induced AP therapy. Administration of insulin is efficient when used to reduce triglyceride levels in patients with HT-induced AP.
\end{abstract}

\section{Introduction}

Acute pancreatitis (AP) is a common condition with several aetiologies. Hypertriglyceridaemia (HT) is a rare but well known cause of AP, which can be a life-threatening complication if the degree of HT is severe enough. Hypertriglyceridaemia as a cause of AP reaches frequencies of $1-7 \%$, according to the literature, when triglyceride levels reach more than $1,000 \mathrm{mg} / \mathrm{dl}$ [1-8]. More than 75\% of HT-induced AP cases are either chronic alcoholics or uncontrolled diabetics [6]. Hypertriglyceridaemia may be primary (e.g. type I, IV, or V) or secondary (diabetes mellitus, alcoholism, pregnancy, obesity, utilisation of some drugs). In most cases that exceed $1500 \mathrm{mg} / \mathrm{dl}$ some form of primary HT or a genetic defeat in lipid metabolism may be seen. Genetic factors are effective in more than $60 \%$ of changes in serum lipids [1, 7, 8]. Apart from conventional methods of treatment, several less frequently used methods have been described in the literature to decrease the tri- glyceride levels rapidly, e.g. plasmapheresis, apoliprotein CII infusion, and use of insulin and heparin $[4,9,10]$. Heparin and insulin are known to stimulate lipoprotein lipase activity [11-13]. The objective of medical treatment is to increase lipoprotein-lipase activity, and to increase chylomicron breakdown, thus diminishing serum triglycerides to levels below 500 or even 200 mg/dl (when possible) using a variety of strategies, including insulin administration. There have been reports in which heparin and insulin have been used for acute reduction of triglycerides, although there are no established guidelines for the efficacy of these modalities. Insulin decreases triglycerides by stimulating lipoprotein lipase activity, which degrades triglycerides into fatty acids and glycerol [4, 10, 11, 13-15].

\section{Aim}

We report 12 cases of HT-induced AP that were successfully treated by insulin. 


\section{Material and methods}

Three hundred and forty-three cases of AP were diagnosed at our clinic between 2005 and 2012. Twelve (3.5\%) of these cases were HT-induced AP. Twelve patients who suffered HT-induced AP are reported. We used "Ranson criteria for non-biliary acute pancreatitis" for assessment of the severity of pancreatitis. Initial blood triglyceride levels were above $1000 \mathrm{mg} / \mathrm{dl}$. Besides the usual treatment of AP, insulin was administered intravenously in continuous infusion. The patients' medical records were retrospectively evaluated in this study.

\section{Results}

Three hundred and forty-three cases of AP were diagnosed and treated at our clinic between 2005 and 2012, and 12 (3.5\%) of these cases were HT-induced AP. Clinical and laboratory parameters of the patients are summarized in Table I. Eight patients were male and four patients were female. The mean age was 48 (35-65) years. The most significant complaint was increase abdominal pain. Nausea and vomiting were observed in most patients. The patients did not have diabetes or alcohol consumption habit. Chest and abdominal X-rays were normal. Cholelithiasis was not detected in abdominal USG. The mean plasma triglyceride level was 1146 (1004-1235) mg/dl, the mean amylase level was 414 (84-780) U/l, and the mean lipase level was 552 (198-1966) U/l. Abdominal computed tomography (CT) was performed for all patients. Patients were diagnosed with AP. According to Ranson criteria, 6 of 12 patients had severe pancreatitis that had a Ranson Severity score more than 3. Eight of the 12 patients were diabetic and others were primary HT. For all patients, oral nutrition was discontinued and conservative treatment was started with intravenous fluid with analgesic and antiemetic administration. Subsequently, patients were started on intravenous regular insulin infusion in 5\% dextrose, making sure that their blood glucose levels were lower than $200 \mathrm{mg} / \mathrm{dl}$. There was an improvement in abdominal pain and nausea-vomiting complaints. Following intravenous insulin treatment, triglyceride (TG) levels decreased to $<500 \mathrm{mg} / \mathrm{dl}$ in 3 days on average, amylase and lipase levels returned to normal levels after 3-4 days, and patients' abdominal pains were resolved. The mean hospitalisation period was 6 days on average [5-9]. Insulin infusions were discontinued after 3 days on average, patients showed clinical improvement, and their plasma TG levels were lower than 300 mg/dl. Patients' treatments were continued using lipid lowering agents following their discharge.

\section{Discussion}

Hypertriglyceridaemia may be responsible for $1-7 \%$ of all cases of $\operatorname{AP}[1,3,4,6,11,13]$. Chylomicronaemia may be responsible for $20 \%$ of AP in non-drinkers free of biliary tract disease. It was previously reported that HT was the cause of $56 \%$ of cases of gestational pancreatitis [16-19]. In many cases, determining the exact aetiology of pancreatitis may be complicated because of the role of ethanol in precipitating severe HT. The proportion of alcoholic pancreatitis caused by direct as opposed to secondary hyperlipidaemic effects is unknown.

Hypertriglyceridaemia-induced AP rarely occurs unless triglyceride levels exceed $1000 \mathrm{mg} / \mathrm{dl}$ [1, 4, 10, 15, 20]. On the contrary, mild to moderate elevations in triglyceride levels are quite common in the early phase of AP of any aetiology. Hypertriglyceridaemia-induced pancreatitis generally occurs when the triglyceride levels exceed $1000 \mathrm{mg} / \mathrm{dl}$, but the exact period that is essential for HT-induced pancreatitis progression is unclear.

More than $75 \%$ of HT-induced patients are either alcoholics or diabetics. Hyperglyceridaemia may be primary (like type I, IV, V hyperlipoproteinaemia) or secondary (like uncontrolled diabetes mellitus), alcoholism, pregnancy, obesity, utilisation of some drugs) [11]. Type I, IV, and $V$ hyperlipoproteinaemia may be seen with AP. The occurrence rate of AP in type I, II and V hyperlipidaemia may be $35 \%, 15 \%$ and $30-40 \%$, respectively [11].

The exact mechanisms of HT-induced pancreatitis are not clear [1, 4]. Chylomicrons are triglyceride-rich lipoprotein particulars that are known to be responsible for inflammation. This condition generally occurs when serum triglyceride levels exceed 1000 mg/dl. Pancreas contains high amounts of lipase. Lipase hydrolyses triglycerides to glycerol and free fatty acids. In normal serum, free fatty acids are bound to albumin and nontoxic. Overloading of lipoproteins may lead to damage in circulatory flow in capillary beds. If this occurs in the pancreas, the resulting ischaemia might damage the acinar structures and expose pancreatic lipase. The generated proinflammatory non-esterified free fatty acids further damage pancreatic acinar cells and microvasculature [16]. Post amplification of the release of inflammatory mediators and free radicals may eventually lead to necrosis, inflammation, oedema, pancreatic ischaemic injury, stasis, slugging of red blood cells, and vascular endothelial damage. This hypothesised sequence of events was proven by studies showing that both triglycerides and free fatty acids caused oedema, haemorrhage, and elevated amylase levels. Hypertriglyceridaemia has also been shown to cause exacerbations in other experimental models of pancreatitis.

Studies using oral lipid-loading tolerance tests have documented elevated past-load plasma triglyceride lev- 


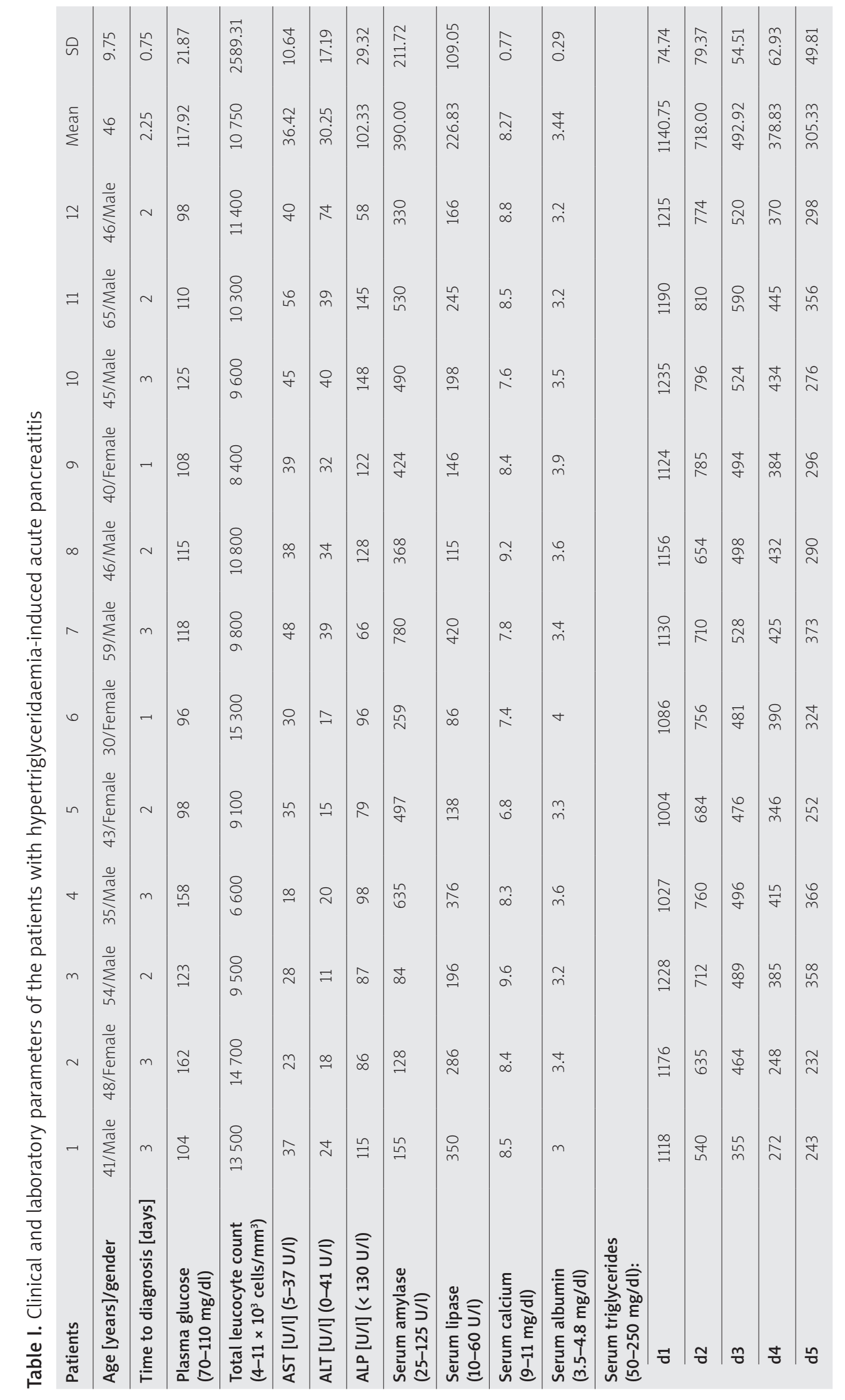


els in patients with previous pancreatitis as compared with control subject. Interestingly, mutations in the lipoprotein lipase (LPL) gene have been identified in patients with HT-induced pancreatitis. Lipoprotein lipase deficiency with chylomicronaemia is a rare recessive disorder characterised by high serum fasting triglyceride levels that may be complicated with AP caused by different LPL gene mutations. normal amylase levels may be incorrectly determined when plasma triglyceride levels exceed $500 \mathrm{mg} / \mathrm{dl}$.

In acute phase HT-induced pancreatitis should be treated in the same manner as other causes of pancreatitis. Currently, there is no clear evidence that HT-induced pancreatitis differs from other types of pancreatitis in terms of frequency of necrosis, complications, or outcomes. A similar approach to medical and diagnostic management is thus indicated.

There are various modalities in the treatment of $\mathrm{HT}$, such as insulin and heparin, plasmapheresis, purified apo C II, and fibric acid derivatives [9, 10, 12, 20, 21].

In an acute setting, direct removal of chylomicrons can be easily done by plasmapheresis. Although plasmapheresis reduces the serum triglyceride levels, it is not a formal therapeutic strategy today.

Lipoprotein lipase is an enzyme that is produced by capillary endothelial cells of muscles and adipose tissues, which hydrolyses the triglycerides to glycerol and fatty acids [11]. Activity of LPL is very important in reducing serum triglyceride levels. Recognising that decreased LPL activity is a prominent cause of HT has fuelled attempts to enhance LPL activity. Heparin and insulin stimulate lipoprotein-lipase activity and accelerate chylomicron degradation [1]. Intravenous insulin and heparin were used in many patients in order to enhance LPL activity and accelerate the chylomicron destruction, and thus are effective in reducing the triglyceride levels rapidly. In several studies it was shown that with insulin and heparin therapy, serum triglyceride levels reduced significantly and pancreatitis improved. In our patients, serum triglyceride levels decreased and pancreatitis symptoms improved in seven days with insulin infusion. Intravenous insulin is an effective and safe method in the therapy of HT-induced AP cases. Diabetic patients should be treated with intravenous insulin infusion in order to obtain and maintain euglycaemia rapidly. Purified apoC-II infusion achieved temporary improvement in triglyceride levels and clinical condition in apoC-II-deficient patients.

Therapeutic effects following recovery from pancreatitis need to be directed at preventing recurrence by controlling triglyceride levels. Secondary HT causes should be treated simultaneously when they exist. Diabetes should be treated with oral hypoglycaemics or insulin in order to maintain strict glycaemic control. If hyperlipidaemia persists despite the fat reduced diet, lipid-lowering agents should be started. The fibric acid derivatives (fibrates) such as gemfibrozil are the drugs of first choice. These agents are generally well tolerated and highly effective if taken regularly and diet restrictions are continued. Also, omega-3 fatty acids, medium-chain triglyceride, and antioxidants may be supplemented.

The limitations of this study are the fact that it was a retrospective study, as well as the small number of cases.

\section{Conclusions}

Our results are compatible with the literature. Insulin may be used safely and effectively in HT-induced AP therapy. Hypertriglyceridaemia is a common clinical problem that can be seen in many medication and medical conditions. A remarkable rise in triglyceride levels may lead to pancreatitis, which is a serious and fatal complication. General and specific therapies are directed at reducing triglyceride levels in the acute phase of pancreatitis. Diet, pharmacological therapy, and avoiding triglyceride-elevating agents are the essential elements of preventing the future attacks. Hypertriglyceridaemia-induced AP covers not only the general characteristics of severe AP but also some specific characteristics. Thus specific strategies should be taken into consideration with the conventional therapy of AP.

\section{Conflict of interest}

The authors declare no conflict of interest.

\section{References}

1. Fortson MR, Freedman SN, Webster PD 3rd. Clinical assessment of hyperlipidemic pancreatitis. Am J Gastroenterol 1995; 90: 2134-9.

2. Jain P, Rai RR, Udawat $H$, et al. Insulin and heparin in treatment of hypertriglyceridemia-induced pancreatitis. World J Gastroenterol 2007; 13: 2642-3.

3. Kota SK, Kota SK, Jammula S, et al. Hypertriglyceridemia-induced recurrent acute pancreatitis: a case-based review. Indian J Endocr Metab 2012; 16: 141-3.

4. Bae JH, Baek SH, Choi HS, et al. Acute pancreatitis due to hypertriglyceridemia: report of 2 cases. Korean J Gastroenterol 2005; 46: 475-80.

5. Berger Z, Quera R, Poniachik J, et al. Heparin and insülin treatment of acute pancreatitis caused by hypertriglyceridemia. Experience of 5 cases. Rev Med Chil 2001; 129: 1373-8.

6. Henzen C, Röck M, Schnieper C, Heer K. Heparin and insulin in the treatment of acute hypertriglyceridemia-induced pancreatitis. Schweiz Med Wochenschr 1999; 129: 1242-8.

7. Gan SI, Edwards AL, Symonds CJ, Beck PL. Hypertriglyceridemia-induced pancreatitis: a case-based review. World J Gastroenterol 2006; 12: 7197-202. 
8. Kozieł D, Kozłowska M, Deneka J, et al. Retrospective analysis of clinical problems concerning acute pancreatitis in one treatment center. Prz Gastroenterol 2013; 8: 320-6.

9. Alagözlü H, Cindoruk M, Karakan T, Unal S. Heparin and insulin in the treatment of hypertriglyceridemia-induced severe acute pancreatitis. Dig Dis Sci 2006; 51: 931-3.

10. Yeh JH, Chen JH, Chiu HC. Plasmapheresis for hyperlipidemic pancreatitis. J Clin Apheresis 2003; 18: 181-5.

11. Serpytis M, Karosas V, Tamosauskas R, et al. Hypertriglyceridemia-induced acute pancreatitiis in pregnancy. J Pancreas 2012; 13: 677-80.

12. Monga A, Arora A, Makkar RP, Gupta AK. Hypertriglyceridemia-induced acute pancreatitis treatment with heparin and insulin. Indian J Gastroenterol 2003; 22: 102-3.

13. Jain D, Zimmerschied J. Heparin and insulin for hypertriglyceridemia-induced pancreatitis: case report. Sci World J 2009; 9: 1230-2.

14. Patel AD. Hypertriglyceridemia-induced acute pancreatitis treatment with insulin and heparin. Indian J Endocr Metab 2012; 16: 671-2.

15. Jimenez Forero SJ, Roa Saavedra DX, Villalba MC. Acute pancreatitis secondary to hypertriglyceridemia: report of two cases. Rev Esp Enferm Dig 2008; 100: 367-71.

16. Yuan G, Al-Shali KZ, Hegele RA. Hypertriglyceridemia: its etiology, effects and treatment. CMAJ 2007; 176: 1113-20.

17. Cappell MS. Acute pancreatitis: etiology, clinical presentation, diagnosis, and therapy. Med Clin North Am 2008; 92: 889-923.

18. Qihui C, Xiping Z, Xianfeng D. Clinical study on acute pancreatitis in pregnancy in 26 cases. Gastroenterol Res Pract 2012; 2012: 271925.

19. Cahalane AM, Smith MJ, Ryan J, Maguire D. Acute pancreatitis secondary to gestational hypertriglyceridaemia. Case Rep Med 2012; 627890.

20. Tsuang W, Navaneethan U, Ruiz L, et al. Hypertriglyceridemic pancreatitis presentation and management. Am J Gastroenterol 2009; 104: 984-91.

21. Kyriakidis AV, Raitsiou B, Sakagianni A, et al. Management of acute severe hyperlipidemic pancreatitis. Digestion 2006; 73: 259-64.

Received: 23.12.2013

Accepted: 1.05 .2014 